\title{
Adding weight to judgments: The role of stimulus focality on weight-related embodied cognition
}

\author{
Leah M. Kaufmann (leah.kaufmann@acu.edu.au) \\ School of Psychology \\ Australian Catholic University, Australia
}

\author{
Sarah Allen (sealle001@myacu.edu.au) \\ School of Psychology \\ Australian Catholic University, Australia
}

\begin{abstract}
Research employing metaphors to explore embodied cognition has shown bidirectional relationships between cognitions and sensory-motor stimuli, such as importance and weight (e.g., "weighty tome"). This research has typically used cognition-relevant metaphor primes (e.g., weighted backpacks when making steepness judgments, weighted clipboards when judging importance of written information) but has yet to consider the role of stimuli features like focality in these findings. The current study examined wearing a heavy versus light backpack on social judgments to explore the effect of this unrelated weight prime on established weight-relevant cognitions. Participants were 40 undergraduate psychology students who wore a heavy $(\sim 5 \mathrm{~kg})$ or light $(<1 \mathrm{~kg})$ backpack while making cognitive, affective, and interpersonal judgments. No significant differences were found between the judgments as a function of backpack weight. This finding suggests that non-task-relevant metaphorical primes have no observable effect on embodied cognition. This provides the first published evidence that embodied cognition is context sensitive and discriminating, that is, not every stimuli activates every related cognition.
\end{abstract}

Keywords: embodied cognition; metaphor; weight; social judgments; body mass index (BMI)

Traditionally, knowledge has been thought to be acquired via modal inputs (e.g., visual and auditory information) which are transformed into abstract amodal representations that can be stored, retrieved, and manipulated (e.g., Pylyshyn, 1973). This view has been challenged by embodied cognition findings that cannot be straightforwardly or efficiently explained by amodal cognition theories. For example, there is substantial evidence for highly localised activation of relevant (e.g., sensory, motor) regions of the brain by abstract constructs (e.g., "lick", "pick", and "kick", differentially activated areas of the brain associated with each specific action; Hauk, Johnsrude, \& Pulvermüller, 2004). This suggests that representations of concepts at least include modal information. Furthermore, there is an advantage for modal-based processing of information as demonstrated in two distinct lines of research. First, perceptual occlusion research has demonstrated that internal features of an object that are not visible in that state are less likely to be identified than when in a visible state (e.g., "seeds" are much more likely to be listed as a feature of "half watermelon" than "watermelon"; Wu \& Barsalou, 2009) suggesting that cognitive processing mimics realworld modal processing. Second, research has found there is a cost associated with information processing that requires a shift between modalities (e.g., making an auditory judgment followed by a visual judgment; Pecher, Zeelenberg, \& Barsalou, 2003).

A final source of support for the embodied cognition perspective is research that has revealed that physical and contextual factor affect seemingly simple cognitions. For example, seminal research by Proffitt and colleagues found that perceptions of the steepness of a slope is consistently over estimated under conditions that would increase the difficulty of traversing it (i.e., viewed atop a hill - Proffitt, Bhalla, Gossweiler, \& Midgett, 1995; wearing a heavy backpack - Bhalla \& Proffitt, 1999; standing on a skateboard atop a hill - Stefanucci, Proffitt, Clore, \& Parekh, 2008). These findings suggest the importance of embodiment in cognition.

In sum, embodied cognition research suggests that cognition is intrinsically and bidirectionally related to the body in which it is occurring. However, this perspective remains somewhat marginal to the more traditional cognitive approach (e.g., Barsalou, 2008; Landau, Meier, \& Keefer, 2010). As a result, much embodied cognition research has focussed on providing examples of embodied cognition, with little work yet to consider the limitations or conditions of embodied cognition effects. For example, it is clear that that physical stimuli influence temporally proximal stimuli (e.g., Ackerman, Nocera, \& Bargh, 2010; Bhalla \& Proffitt, 1999; Slawuta \& Castano, 2011). However, there is no evidence to suggest that physical stimuli have long term or distal effects. Thus, it is likely that there are temporal limitations to embodied cognition effects. Other limitations may include relevance (i.e., stimuli that have no relationship to the cognition are 
likely to have little or no effect) and focality (i.e., stimuli that are wholly unnoticed or undetected are likely to have an effect on cognition), which would ensure embodied cognitions are usefully discriminating (i.e., affected only by key factors). By understanding the scope and limits of embodied cognitions it will be possible to develop a nuanced and sophisticated understanding of the interplay between cognition and embodiment.

\section{Metaphors in Embodied Cognition}

Metaphors have been used in embodied cognition research as tools for understanding the link between abstract, often social concepts (e.g., interpersonal warmth or closeness) and physical stimuli (e.g., a warm drink). These findings have provided a theoretical basis for addressing the greatest weakness of embodied cognition theory, namely, how abstract concepts (e.g., love) are embodied in terms of modalities (e.g., haptic warmth).

Weight is a physical property that is linked to several metaphorical concepts. Common weight metaphors include "emotional weight" and "weighing up", which link the physical property of weight to the abstract concepts of importance, seriousness, or burden, and evaluation. Embodied cognition research exploring metaphorical weight has typically used a procedure in which participants hold a heavy or light object before making judgments regarding metaphorically related concepts (e.g., importance, seriousness). For example, Jostmann, Lakens, and Schubert (2009) found that participants who held a heavy clipboard $(1.04 \mathrm{~kg})$ placed a higher value on a foreign currency than did those holding a light clipboard $(0.66 \mathrm{~kg})$. In a second study, participants holding the heavy clipboard rated having their opinion heard as more important than did those holding a light clipboard. Interestingly, participants holding the heavy clipboard had significantly greater confidence in their own arguments compared to those holding the light clipboard.

A similar approach was used by Ackerman, Nocera, and Bargh (2010) who found that participants who held a heavy clipboard $(2.04 \mathrm{~kg})$ rated a job applicant to be more serious, and their own judgment about the applicant to be more important than participants who held the light clipboard $(0.30 \mathrm{~kg})$. The clipboard weight did not, however, have any significant effect on applicant's likeability, which was interpreted as evidence that weight uniquely affect importance- or seriousness-related cognitions. Kaspar (2013) used the heavy $(1.61 \mathrm{~kg})$ versus light $(0.22 \mathrm{~kg})$ clipboard manipulation to examine the effect of physical weight on the perceived seriousness of diseases, drug treatment side-effects, and drug treatment effectiveness. Study 1 revealed that participants who held the heavy clipboard rated both diseases and drug side effects as significantly more serious than those who held the light clipboard. Study 2 revealed that, while clipboard weight had no effect of estimated recovery time, participants who held the heavy clipboard rated the drug treatment as significantly more effective than those who held the light clipboard. Finally, weighted clipboards (heavy = $1.67 \mathrm{~kg}$; light $=0.35 \mathrm{~kg}$ ) were found to influence the effectiveness but not the side-effect severity of general drug treatment (Study 3) or weight-reduction drugs (Study 5). Interestingly, no relationship was found between these ratings which were interpreted as evidence for limited cognitive-based consistency of embodied cognitions resulting from the non-conscious nature of the manipulation.

In sum, these findings consistently demonstrate that physical weight, as manipulated by a weighted clipboard, significantly affects the judgments of seriousness or importance across a range of topics. It should be noted that, in each case, the physical stimuli was not relevant to the ratings (i.e., there is no reason for the weight of a clipboard on which ratings are recorded to be related to the content of the ratings), however, the stimuli was focal in each experiment. That is, participants were aware of holding a clipboard. Likewise, in the only study exploring metaphorical weight that did not use the clipboard manipulation (e.g., Kaspar, 2013 also used weighted packages of drug treatments including a heavy light package but had no effect on side-effect severity) also revealed a consistent result (i.e., heavy packages were associated with higher effectiveness ratings), again using a focal stimuli. Consequently, it can be confidently concluded that weight affects metaphorically related judgments (e.g., importance, seriousness), however, it is unclear if this effect is limited to focal stimuli, or whether it is a more general effect (i.e., non-focal stimuli have a similar effect)..

The bidirectionality of metaphorical embodied cognitions is considered a strength of this literature, and well-established as part of the metaphorical weight research. For example, reversing the typical procedure, Schneider, Rutjens, Jostmann, and Lakens (2011) found that a textbook described as "important" was rated nearly $50 \%$ heavier than a "textbook" about which no importance information was given. In a second study, participants who estimated the weight of an important textbook reported it was significantly heavier than those who rated a textbook that was effortful to read, or a textbook. Moreover, this effect was stronger for those who held the important textbook compared to those who only looked at it, suggesting the effect was exaggerated by the involvement of the relevant (i.e., haptic) modality.

Exploring the association between weight metaphorical burden, Slawuta and Castano (2011) found that page on which a shameful memory is described is estimated to be significantly heavier than a page on which a neutral memory is described. Similarly, a page on which stories of in-group misdeeds are described is significantly heavier than a page on 
which out-group misdeeds are described. This finding suggests that only self-relevant psychological burdens affect perceived physical weight. However, Susewind's (2012) finding that participants who evaluated unethically produced chocolate bars estimated them to be heavier than those who evaluated ethically produced chocolate bars, suggests that personal responsibility may not be essential to this effect (i.e., the participants were not responsible for the unethical production). Rather, perhaps these effects are driven be selfassociation instead of responsibility as participants in Susewind's experiment were instructed that they would consume the chocolate they evaluated, increasing the personal relevance of the other-produced chocolate.

These findings, like the many that have demonstrated evidence for a bidirectional perception-cognition link through the use of metaphors (e.g., Lakoff \& Johnson, 1980; Leander, Chartrand, \& Bargh, 2013; Lee \& Schwarz, 2012; Meier, Robinson, \& Clore, 2004; Sherman \& Clore, 2009; Slepian \& Ambady, 2012) provide further support for embodied cognition. However, it is noteworthy that this research has exclusively used focal or relevant stimuli. That is, each procedure has had participants actively engage with the manipulation stimuli (e.g., focal manipulation: hold the weighted clipboard while making ratings on it) or consider the stimuli as the basis for decision (e.g., rate and estimate the weight of an important textbook) and observed the effect on cognitions. Consequently, these findings provide evidence for an effect of physical weight on metaphorically relevant cognitions (e.g., importance, burden), however, only under conditions that the weight is focal, or relevant. Thus, to provide support for the general conclusion (i.e., a link between weight and importance), it is essential to demonstrate that this effect also holds for non-focal and non-relevant weight.

\section{The Current Study}

The current study was designed specifically to explore the effect of a non-focal, non-relevant physical weight on metaphorical importance or seriousness. For this reason, a weighted backpack, which has been found to be an effective weight manipulation in previous research (e.g., Proffitt et al., 2003) was used in this study as a non-focal and non-relevant manipulation of physical weight. Participants were randomly assigned to either a heavy $(\sim 5 \mathrm{~kg})$ or light $(\sim 1 \mathrm{~kg})$ backpack condition under the guise of being asked to wear an "accessory" (e.g., sunglasses, gloves) before completing measures assessing the seriousness of and compensation for a property crime, rated the emotion and psychological burden of a guilty memory, and judged the funniness of and light-heartedness having read five jokes. We argue that this manipulation is not a focal manipulation because the backpack plays no role in any other aspect of the procedure (e.g., is it not used in ratings) and is not relevant to any judgment or rating (i.e., backpacks are not related to importance, guilt, funniness, etc.).

Consistent with the weight metaphors, we expect that participants primed with a heavy versus light physical weight would be more likely to experience and report greater seriousness, burden, and a more sombre outlook. Consequently, it was predicted that participants wearing a heavy backpack would rate the crimes as more serious, report feeling more psychological burden (e.g., guilt), and report lower levels of amusement and light-heartedness compared to those wearing a light backpack. These findings would provide evidence of a general effect of physical weight on embodied cognition via the metaphor of importance or seriousness. Conversely, the absence of such effects would suggest that embodied cognitions are context sensitive and discriminating so that only focal or relevant stimuli have such an influence.

\section{Method}

\section{Participants}

The sample size was based on previous research (e.g., Ackerman et al., 2010; Jostmann et al., 2009; Proffitt et al., 2003; Slepian, Masicampo, \& Ambady, 2014; Witt, Proffitt \& Epstein, 2004) which has demonstrated large ( 0.7) effect sizes (Cohen, 1992), which suggests a sample of 25-43 participants would be adequate to detect the predicted differences (Brant, 2012). Consequently, participants were 40 people recruited from the Melbourne campus of Australian Catholic University and from a sample of convenience. Of the 40 participants, seven were male and 33 were female $\left(M_{\text {age }}=30.10, \quad S D_{\text {age }}=13.83\right)$. Student participants received $1 \%$ course credit in a psychology unit for their participation in this study.

\section{Materials}

Manipulation. A backpack was filled with either a $5 \mathrm{~kg}$ bag bread mix (i.e., heavy back pack condition) or two $160 \mathrm{~g}$ packets of cotton balls (i.e., light backpack condition).

Participant variables. Participants' age and gender were recorded. In addition, participants' Body Mass Index (BMI) was assessed using a weighing scale and height chart.

Seriousness. Two short vignettes were developed for the purpose of the experiment. Each described an incident in which a person recklessly damaged property and was apprehended for the crime. The first vignette also described the financial compensation awarded by a judge against the perpetrator. For the second vignette, participants were asked to assign a monetary compensation against the perpetrator. Participants were 
then asked to rate the seriousness of the crime on a scale from 1 (not at all serious) to 7 (very serious), and the consideration necessary to allocate financial compensation on a scale from 1 (very little consideration) to 7 (a lot of consideration). Finally, for the first vignette, participants rated the appropriateness of the financial compensation awarded to the victim of the crime by the judge on a scale from 1 (not at all appropriate) to 7 (very appropriate), and for the second vignette, what compensation they would award the victim from $1(\$ 1,000)$ to $7(\$ 10,000)$, and how difficult it was to make a compensation decision on a scale from 1 (not at all difficult) to 7 (very difficult). Scores for seriousness and appropriateness were calculated by averaging ratings of the two vignettes. Compensation appropriateness, compensation values, and judgment difficulty were used as single item measures.

Psychological burden. Participants were asked to think of a time when they had acted in a way they were not proud of. Participants were then asked to write down 5 words, which reflected that memory on a single A4 page. Participants were advised that this piece of paper was not going to be viewed or kept by the experimenter, and that participants would be provided with the opportunity to securely dispose of this paper at the end of the experiment. Participants then provided ratings of how they felt about this memory in terms of negativity from 1 (very negative) to 7 (very positive), guilt 1 (very guilty) to 7 (not at all guilty), shame 1 (very ashamed) to 7 (not at all ashamed), and sadness 1 (very sad) to 7 (very happy). Finally, participants also rated the degree to which the memory "weighed on" them on a 7 point scale from 1 (does not weigh on me at all) to 7 (weighs on me very heavily). The negativity, guilt, shame, and sadness items were averaged to form a general negative affect scale which demonstrated good internal consistency $(\alpha=.83)$. The item, "weighs on me" was used as a single item rating of perceived psychological burden. Finally, replicating Slawuta and Castano (2011), participants were asked as part of a purportedly separate task to estimate the weight (in $\mathrm{mg}$ ) of the sheet of paper on which the memory was described, as well as two other objects a butterfly and a feather.

Light-heartedness. Participants read five jokes (e.g., "I used to own a paper shop. It blew away") rated as highly funny in an unrelated study (de la Piedad Garcia \& Kenny, 2011). Participants rated the funniness of each joke separately on a 7 point scale from 1 (not at all funny) to 7 (very funny). Participants were also indicated how light-hearted they felt after reading the jokes on a 7 point scale from 1 (not at all light hearted) to 7 (very light-hearted). The funniness and lightheartedness ratings were averaged to create a measures of light-heartedness which demonstrated adequate internal consistency $(\alpha=.74)$.

\section{Procedure}

Participants attended individual participation sessions on campus. On arrival, participants were presented with an information letter describing the topic of the study as an examination of the effect of "accessories on cognition" which was used to obscure the true purpose of the study (i.e., weight). To maintain the pretence of the study, the backpack was placed among other accessories (e.g., sunglasses, a scarf, hat, and gloves) and seemingly randomly assigned to the participant by the experimenter at the beginning of participation. The backpack was always pre-filled with the heavy or light contents prior to the participant's arrival.

To ensure participants experienced the full effect of the backpack weight, all measures were completed at standing stations located around the experiment room, requiring the participant to walk between stations and to complete measures while standing. The participants were then required to complete some demographic information (e.g., age, gender, and place of birth) and were assigned an accessory. The participants began by providing demographic information, before completing the seriousness, psychological burden, and lightheartedness measures in an order determined by a Latin Square Design to ensure that order effects were minimised. Finally, participants were asked to estimate the weight of the backpack, and their own height and weight were measured, before the participant completed a funnel debriefing to check for any awareness of the true purpose of the study. Only one participant mentioned the backpack specifically during the funnel debriefing, but did not report any suspicion about the role of the backpack weight in this study. On this basis, no participants were excluded from the study. Participation was completed in a single session which took approximately 30 minutes.

\section{Results}

\section{Descriptive statistics}

The descriptive statistics (see Table 1) for key variables as a function of backpack condition revealed that participants in the heavy backpack condition rated the backpack as heavier than those in the light backpack condition. As can be seen no other differences were large, and only negative affect, psychological burden, and light-heartedness were in the predicted direction (see Table 1). 
Table 1: Means and Standard Deviations for BMI, Seriousness, Psychological Burden, and Light-heartedness Variables as a Function of Backpack Condition

\begin{tabular}{|c|c|c|c|c|}
\hline & \multicolumn{2}{|c|}{ Heavy backpack $(n=20)$} & \multicolumn{2}{|c|}{ Light backpack $(n=20)$} \\
\hline & $M$ & $S D$ & $M$ & $S D$ \\
\hline Backpack weight & 7.76 & 5.20 & 1.22 & 1.16 \\
\hline BMI & 23.32 & 4.22 & 23.88 & 5.40 \\
\hline \multicolumn{5}{|l|}{ Seriousness } \\
\hline Seriousness rating & 3.85 & 0.88 & 4.30 & 1.26 \\
\hline $\begin{array}{l}\text { Consideration } \\
\text { rating }\end{array}$ & 4.02 & 0.69 & 3.93 & 0.96 \\
\hline $\begin{array}{l}\text { Compensation } \\
\text { appropriateness } \\
\text { (vignette 1) }\end{array}$ & 4.30 & 1.30 & 4.70 & 1.50 \\
\hline $\begin{array}{l}\text { Compensation } \\
\text { value (vignette 2) }\end{array}$ & 4200.00 & 2160.65 & 5775.00 & 2876.74 \\
\hline $\begin{array}{l}\text { Judgment } \\
\text { difficulty } \\
\text { (vignette 2) }\end{array}$ & 3.80 & 1.28 & 3.95 & 1.54 \\
\hline \multicolumn{5}{|l|}{$\begin{array}{l}\text { Psychological } \\
\text { Burden }\end{array}$} \\
\hline Negative affect & 2.73 & 0.84 & 2.50 & 0.94 \\
\hline $\begin{array}{l}\text { Psychological } \\
\text { burden rating }\end{array}$ & 4.20 & 1.58 & 3.75 & 1.37 \\
\hline $\begin{array}{l}\text { Paper weight } \\
\text { (mg) }\end{array}$ & 21.99 & 40.13 & 30.66 & 43.17 \\
\hline \multicolumn{5}{|l|}{ Light-heartedness } \\
\hline $\begin{array}{l}\text { Light-heartedness } \\
\text { rating }\end{array}$ & 3.76 & 1.09 & 4.04 & 0.86 \\
\hline
\end{tabular}

\section{Analyses}

Manipulation check. An independent samples t-test was used to examine participants' perception of backpack weight and revealed participants in the heavy backpack condition rated their backpack as significantly heavier than those in the light backpack condition $(t(38)=5.48, p<.01,95 \%$ CI $[2.90,6.08])$. Correlations between estimated backpack weight and all the variables of interest were not significant (all $p$ 's $>.51$ ).

BMI. BMI was included as a potential weightrelevant unintentional confound or covariate. For this reason, significant differences in participant's BMI as a function of backpack condition was examined using an independent samples t-test and revealed no significant difference $(t(38)=0.37, p=.72,95 \%$ CI $[22.07,25.13])$. In addition, correlations between BMI and all variables of interest were examined to investigate potential covariation. Results revealed BMI was found to significantly correlate with seriousness rating $(r(40)=.41, p=.008)$, consideration rating $(r(40)=.35$, $p=.03)$, and judgment difficulty $(r(40)=.34, p=.03)$. No other significant correlations were found.

Seriousness. Independent samples t-tests were used to examine the seriousness ratings, consideration, compensation appropriateness, compensation values, and judgment difficulty as a function backpack condition. No significant differences were found for seriousness rating $(t(38)=1.31, p=.20,95 \% \quad \mathrm{CI}$
$[3.72,4.43])$, consideration given $(t(38)=0.32, p=.75$, 95\% CI [3.71,4.24]), compensation appropriateness $(t(38)=0.92, \quad p=.36, \quad 95 \% \quad$ CI $\quad[4.06,4.94]), \quad$ or compensation values $(t(38)=1.96, p=.06,95 \%$ CI [4114.85,5830.15]) as a function of backpack condition.

Psychological Burden. Independent samples t-tests were used to examine the experience of negative affect and the psychological burden of a memory of which participants were not proud, as well as the weight of a page on which the memory was written. A trend in the predicted direction was found for negative affect and psychological burden (see Table 1), however, neither was found to differ significantly as a function of backpack condition $\left(t_{\text {negative affect }}(38)=0.80, p=.43,95 \%\right.$ CI $[2.33,2.90])$ and $\left(t_{\text {psychological burden }}(38)=0.96, p=.34\right.$, $95 \%$ CI $[3.43,4.32])$. Similarly, no significant difference was found in the estimated weight of the paper on which the negative event was briefly described as a function of backpack condition $(t(38)=0.66, p=.51$, $95 \%$ CI $[13.09,39.55])$

Light-heartedness. An independent samples t-test was used to examine differences in light-heartedness ratings as a function of backpack condition. Consistent with predictions, participants in the heavy backpack condition reported lower levels of light-heartedness than did participants in the light backpack condition (see Table 1), however, this difference did not reach significance $(t(38)=.091, p=.37,95 \%$ CI $[3.59,4.21])$.

\section{Discussion}

The results revealed no significant effect of nonfocal, non-relevant (i.e., backpack) weight on judgments of the seriousness of a crime, or on compensation awarded; on the emotion or psychological burden of a guilty memory; or on the funniness of five jokes and the consequent lightheartedness. However, a finding that BMI was correlated with seriousness judgments (i.e., seriousness rating, consideration rating, and judgment difficulty) suggests there may be habitual effects of embodiment. For example, people with higher BMI made more extreme judgments, but also gave these judgments more consideration, and reported greater difficulty in making these judgments than did people with lower BMI. Future research is required to examine this, as yet, largely unexplored topic of the effect of one's own body on embodied cognitions.

The findings of the current study indicate that embodied cognitions are not indiscriminately affected by physical or contextual stimuli. Specifically, the nonfocal, non-relevant weighted backpack produced small, non-significant effects. This is in contrast to previous findings which, having used focal (e.g., a clipboard used in making judgments) or relevant physical stimulus primes (e.g., the package of drugs being 
evaluated, or the page on which a negative memory is described) found large effects of weight on importanceor seriousness-judgments. As a result, this is not a failure to replicate previous research (e.g., Ackerman et al., 2010; Jostmann et al., 2009, Slawuta \& Castano, 2011) but rather suggests that these effects are likely to have sensible limits. Future research is, however, needed to directly compare the effects of a focal nonrelevant stimuli (e.g., clipboard, replicating previous finding) and a non-focal non-relevant stimuli (e.g., a backpack replicating the current finding) in a single study before such a conclusion can be unequivocally drawn.

A limitation of the current research is that a small, homogenous sample and largely female sample, however, as there was no basis for gender-specific effects, this last issue is of limited concern in the current research. As already discussed, this feature of the study design was based on previous research, which has consistently found large effects that are detectable with samples of this size (e.g., Ackerman et al., 2010; Jostmann et al., 2009). Furthermore, a post-hoc examination of effect sizes obtained in the current study suggests that the influence of a non-focal, non-relevant weight prime on the weight-importance or -seriousness cognition is, in contrast, small to very small. As a result, the required sample size to detect a significant effect using this manipulation well exceeds those previously published (i.e., estimates ranged from $n=82$ to $n=2640$; Brant, 2012). Moreover, as effect size is not dependent on sample size, the findings of the current study which found that the current manipulation had no detectable effect on metaphorically (i.e., weight) related embodied cognitions is consistent with the proposition that embodied cognitions are likely to be appropriately limited. Future research examining the range and limits of embodied cognition should include both relevant and non-relevant primes to allow direct comparison of effect sizes within a single study.

The unexpected finding that BMI was significantly correlated with seriousness judgments suggests bodily states as a new topic for research in embodied cognition. For example, it is possible that individuals with higher BMI (i.e., heavier) may be more prone to judgments that a situation, action, or outcome is serious, which may have important implications (e.g., legal rulings, funding recommendations). Moreover, this suggests that own weight (e.g., BMI) may be chronically focal, affecting previously unconsidered cognitions such as seemingly irrelevant seriousness judgments. Future research should explore the potential implication of this finding as the basis for habitual overor under-estimation of seriousness and risk-taking, which may be easily affected by a simple change in an individual's physical state.

One interesting implication of these findings is that it is possible that stimuli relevance may, in fact, change with context or time so that its influence is not always observable but may, under some very specific circumstances, dominate cognitions (i.e., stimuli relevance reflects not only the properties of the stimuli, but also the person in temporal and physical context). Such a finding would demonstrate significant maturity of this field, and would be a significant development in the topic of embodied cognition.

Another important applied implication of the current findings is that ratings of importance, seriousness or burden are unlikely to be influenced by physical weight so long as these are non-focal or non-relevant to a situation. For example, ratings should be collected a station (e.g., table) rather than using a hand-held clipboard to avoid introducing a weight (i.e., importance) prime.

In conclusion, the current study suggests that the weight-importance or weight-seriousness link is not elicited by non-focal or irrelevant weight stimuli. This is interpreted as evidence for the discriminating nature of embodied cognitions. That is, influences on embodied cognition seem to be limited to physical and contextual factors that are relevant (e.g., conceptually, temporally). This allows such a system to limit competition between the many potential influences (e.g., a hot beverage in the hand versus a cold day outside) the result of which is that situationally appropriate and possibly beneficial effects would be most likely (e.g., interpersonal warmth towards the cup bearer).

\section{References}

Ackerman, J. M., Nocera, C. C., \& Bargh, J. A. (2010). Incidental haptic sensations influence social judgments and decisions. Science, 328, 1712-1715. doi:10.1126/science.1189993

Barsalou, L. W. (2008). Grounded cognition. Annual Review of Psychology, 59, 617-645. doi:10.1146/annuev.psych.59.103006.093639

Bhalla, M., \& Proffitt, D. R. (1999). Visual-motor recalibration in geographical slant perception. Journal of Experimental Psychology: Human Perception and Performance, 25, 1076-1096. doi:10.1037/0096-1523.25.4.1076

Brant, R. (2012). Inference for a mean: Comparing a mean to a known value [Online power calculator]. Retrieved from: http://www.stat.ubc.ca/ rollin/stats/ssize/n1.html

Cohen, J. (1992). A power primer. Psychological Bulletin, 112, 155-159. doi:10.1037/00332909.112.1.155

Connell, L., \& Lynott, D. (2011). Modality switching costs emerge in concept creation as well as retrieval. Cognitive Science, 35, 763-778. doi: 10.1111/j.15516709.2010.01168.x

de la Piedad Garcia, X., \& Kenny, T. (2011). Cognitive load or valence? The effect of humour on negative emotions. Paper presented at the European 
Association of Social Psychology conference, Stockholm, Sweden.

Hauk, O., Johnsrude, I., \& Pulvermüller, F. (2004). Somatotopic representation of action words in human motor and premotor cortex. Neuron, 41, 301-307. doi:10.1016/S0896-6273(03)00838-9

Horton, W. S., \& Rapp, D. N. (2003). Out of sight, out of mind: Occlusion and the accessibility of information in narrative comprehension. Psychonomic Bulletin and Review, 10, 104-110.

Jostmann, N., Lakens, D., \& Schubert, T. W. (2009). Weight as an embodiment of importance. Psychological Science, 20, 1169-1174. doi:10.1111/j.1467-9280.2009.02426.x

Kaspar, K. (2013).A weighty matter: Heaviness influences the evaluation of disease severity, drug effectiveness, and side effects. PlosOne, 8, 1-9. doi:10.1371/journal.pone. 0078307

Lakoff, G. \& Johnson, M. (1980). The metaphorical structure of the human conceptual system. Cognitive Science, $\quad 4, \quad 195-208$. doi:10.1207/s15516709cog0402_4

Landau, M. J., Meier, B. P., \& Keefer, Lucas, A. (2010). A metaphor-enriched social cognition. Psychological Bulletin, 136, 1045-1067. doi:10.1037/a0020970

Leander, N. P., Chartrand, T. L., \& Bargh, J. A. (2012). You give me the chills: Embodied reactions to inappropriate amounts of behavioural mimicry. Psychological Science, $18, \quad 772-779$. doi: $10.1177 / 0956797611434535$

Lee, S. W., \& Schwarz, N. (2012). Bidirectionality, mediation, and moderation of metaphorical effects: The embodiment of social suspicion and fishy smells. Journal of Personality and Social Psychology, 103, 737-749. doi:10.1037/a0029708

Meier, B. P., Robinson, M. D., \& Clore, G. L. (2004). Why good guys wear white: Automatic inferences about stimulus valence based on brightness. Psychological Science, $\quad 15, \quad 82-87$. doi:10.1111/j.0963-7214.2004.01502002.x

Pecher, D., Zeelenberg, R., \& Barsalou, L. W. (2004). Sensorimotor simulations underlie conceptual representations: Modality-specific effects of prior activation. Psychonomic Bulletin \& Review, 11, 164167. doi:10.3758/BF03206477

Proffitt, D. R., Bhalla, M., Gossweiler, R., \& Midgett, J. (1995). Perceiving geographical slant. Psychonomic Bulletin and Review, 2, 409-428.

Pylyshyn, Z. W. (1973). What the mind's eye tells the mind's brain: A critique of mental imagery. Psychological bulletin, 80, 1-24.

Schneider, I. K., Rutjens, B. T., Jostmannm N. B., \& Lakens, D. (2011). Weighty matters: Importance literally feels heavy. Social Psychological and Personality Science, 2, 474-478. doi:10.1177/1948550610397895
Sherman, G. D., \& Clore, G. L. (2009). The color of sin: White and black are perceptual symbols of moral purity and pollution. Psychological Science, 20, 1019-1025. doi:10.1111/j1467-9280.2009.02403.x

Slawuta, P., \& Castano, E. (2011). The unbearable heaviness of sin. Paper presented at the European Association of Social Psychology conference, Stockholm, Sweden.

Slepian, M. L., \& Ambady, N. (2012). Fluid movement and creativity. Journal of Experimental Psychology: General, 141, 625-629. doi:10.1037/a0027395

Slepian, M. L., Masicampo, E. J., \& Ambady, N. (2014). Relieving the burdens of secrecy: Revealing secrets influences judgments of hill slant and distance. Social Psychological and Personality Science, 5, 293-300. doi:10.1177/1948550613498516

Stefanucci, J., Proffitt, D. R., Clore, G. L., \& Parekh, N. (2008). Skating down a steeper slope: Fear influences the perception of geographical slant. Perception, 37, 321-323. doi:10.1068/p5796

Susewind, M. (2012). Subjective morality - Empirical studies on how people balance their own interests with the interests of others and experiencing moral meaning. (Unpublished doctoral dissertation). University of Cologne, Cologne, Germany.

Witt, J. K., Proffitt, D. R., \& Epstein, W. (2005). Tool use affects perceived distance, but only when you intend to use it. Journal of Experimental Psychology: Human Perception and Performance, 31, 880-888. doi:10.1037/0096-1523.31.5.880

Wu, L. L., \& Barsalou, L. W. (2009). Perceptual simulation in conceptual combination: Evidence from property generation. Acta Psychologica, 132, 173189. doi:10.1016/j.actpsy.2009.02.002

\section{Correspondence to:}

Dr Leah M. Kaufmann

School of Psychology

Australian Catholic University

Melbourne Campus (St Patrick),

Locked Bag 4115,

Victoria 3065,

email: leah.kaufmann@acu.edu.au

\section{Research Profile}

Dr Leah Kaufmann is a lecturer and early career researcher at Australian Catholic University in Melbourne, Australia. Her research interests are implicit social cognition and embodied cognition. Leah's research has principally focused on the development and use of implicit methods (e.g., priming, GNAT) for understanding intergroup topics such as prejudice with specific interests in implicit racism, implicit homophobia, and implicit sexism. More recently, she has extended this research to explore selfrelevant cognitions in health behaviours (e.g., implicit body image and its relationship to unhealthy eating. Leah has also undertaken several projects with student 
Kaufmann \& Allen: Adding weight to judgments

researchers exploring the effects of embodied cognition as an unconscious influence on behaviour. 